\title{
SE L'ANTIMAFIA È DONNA. IL POTERE DELLA PAROLA FEMMINILE NELL'AFFERMAZIONE DI UNA CULTURA DELLA LEGALITÀ
}

\author{
Irene Strazzeri \\ Università degli Studi di Foggia (IT)
}

http://dx.doi.org/10.5209/NOMA.53540

\begin{abstract}
IT).-La presenteindagine si propone di dimostrare quanto l'antimafia sia intessuta di soggettività politiche femminili e quanto l'esperienza femminile, maturata in seno ad associazioni, che fin dagli inizi hanno interpretato e combattuto le mafie adottando un'ottica antigiustizialista, improntata all'antimafia sociale e culturale, abbia ridisegnato con linguaggi creativi un nuovo immaginario dell'antimafia. Ciò è possibile coltivando la passione per la ricerca e la pratica di un sapere altro: quello di genere. I testi e le ricerche utilizzati in questo articolo provengono in gran parte dagli studi di genere e dalla cultura femminista, che ha modificato lo sguardo sulle tante battaglie per affermare la cultura della legalità.
\end{abstract}

Parole chiave: Genere, racconto, anti-mafia, cultura della legalità.

Abstract.-Thissurveyaims to show how Anti-mafia isinterwoven with femalepoliticalsubjectivity and howfemaleexperience, gained byassociations, in which from beginningplayed a role a perspectivebased on legal culture, havecreateda new Anti-mafia imaginary. Thisispossible by adoptinggenderperspective in researchandknowledge. Articles and researchused in thisreflection come largely from gender studies and feminist culture, whichchanged the look on manystruggles to affirm a culture of legality.

Keywords: Gender, Narration, Anti-mafia, Culture of Legality.

\section{Introduzione}

Un fil rouge unisce due ordini discorsivi in questa riflessione. In primo luogo lo sguardo di genere: uno sguardo che fa la differenza sulla lettura dei mutamenti sociali e culturali che coinvolgono i soggetti, le relazioni di genere e la società intera(Siebert, 1994); in secondo luogo l'importanza del racconto come atto di civiltà politica(Dino, 2002), valore che rimanda a quella cura per il bene comune e per sentimenti, segno di immutabilità ed eternità contro l'azione mortifera e violenta delle mafie. Raccontare significa assumersi la responsabilità di dare visibilità alle cose che accadono, ignorate e strumentalizzate, agendo quel passaggio dall'antropologia del "non mi riguarda", retaggio diarcaismi mafiosi e culturali riattualizzati dalla criminalità organizzata, a quella del "ci riguarda", che è oggi la grammatica sentimentale di molte esperienze politiche. II racconto è il potere di strappare all'oblio la realtà delle cose, sfidare l'egemonia delle narrazioni convenzionali, superando interpretazioni stereotipate e vuoti di memoria che godono spesso di complicità istituzionali. Raccontare è infatti opera di svelamento. È questo il senso dei molti lavori che hanno segnato la storia dell'antimafia: il libro "Dimenticati" di Danilo Chirico e Alessio Magro, che racconta da un punto di vista inedito la storia delle donne e degli uomini uccisi dalla 'ndrangheta e dimenticati dallo Stato (2010); "Roberta Lanzino, Ragazza" di Celeste Costantino, il primo fumetto italiano che racconta la storia di un femminicidio con implicazioni mafiose (2012).

Il tema delle mafie che uccidono le donne è stato affrontato in modo parziale dalle scienze sociali, lasciando sullo sfondo molte questioni riguardanti il rapporto donne e potere, la "femminilizzazione della società", per citare una categoria attraverso cui leggere le contraddizioni implicate nel processo di emancipazione femminile. 
Innanzitutto sarebbe opportuno raccontare le storie, più o meno emblematiche, delle donne coinvolte nelle organizzazioni criminali, collocandole di volta in volta in tre esperienze differenti: ci sono le "donne contro", le "donne di mezzo" e le "donne di potere", ad esempio (Madeo, 1994). Suddivisione che certamente non risponde ad una specifica categoria di donne coinvolte nei processi mafiosi, quanto piuttosto alla necessità di articolare le problematiche di cui le protagoniste sono espressione, a partire dal loro posizionamento nell'ambiente mafioso e dalle scelte differenti che hanno sostenuto.

Ponendo al centro le storie di vita delle donne si può dimostrare quanto le loro singole biografie, sebbene non rimandino ad un discorso universale sul ruolo femminile nelle mafie, scivolino su questioni che si affacciano sul presente portandoci ad una traccia comune: il rapporto donne e potere, la tensione tra emancipazione e libertà femminile, la dinamica tra assimilazione ed estraneità che definisce la soggettività femminile, erede di una memoria storica di sudditanza, ma anche di una forza che rompe gli argini, scardina gerarchie di potere, riscrive i destini da vittime a soggetti pieni. $\dot{E}$ ciò che accomuna le donne che hanno sfidato la mafia, raccontate nel libro curato da Cinzia Paolillo. "Donne contro"(Incandela, 2007), che nella maggior parte dei casi, a seguito all'uccisione violenta dei propri congiunti per fatti di mafia, hanno trasformato il dolore privato del lutto in una scelta civica di protesta e ribellione. "Donne contro" sono le donne che si stanno impegnando nella lotta alla mafia per motivi diversi, alcuni dei quali non necessariamente collegati ad elementi biografici. Protagoniste di un medesimo gesto di rottura sono le "donne di mezzo", ossia quelle donne che provengono da contesti mafiosi nei quali sono nate e cresciute o con cui sono venute successivamente in contatto. Sono donne di confine tra un "dentro", di cui hanno fatto parte anche assumendo ruoli attivi nell'attività criminale, e un "fuori" che, in seguito alla scelta di collaborare, è tutto da riempire e reinventare attraverso nuovi valori di riferimento. II viaggio interiore che devono attraversare nelle loro esistenze segnate da condizionamenti culturali maschilisti e mafiosi, prevaricate da storie di violenza di genere, diviene una sfida da reinventare senza modelli di riferimento cui ispirarsi o da imitare (Siebert, op. cit. 1994). Un altro spaccato, infine, è proposto dalle donne che assumono un ruolo di comando nelle organizzazioni criminali. In questo caso i racconti offrono un quadro interessante, entro cui analizzare il protagonismo femminile nell'attività criminale, il dilemma tutto da indagare dell'emancipazione femminile, che molte studiose hanno definito "ambigua"(A.A.V.V., 1996), espressione che permette di fotografare le "donne al potere", considerando i limiti di questo fenomeno, analizzando le contraddizioni prodotte dai cambiamenti sociali, così come i condizionamenti che hanno portato le donne a compiere simili scelte.

Tra coloro che hanno privilegiato lo sguardo di chi ha indagato e pensato le mafie in maniera profonda e orientata al genere, vanno sicuramente ricordatiNando dalla Chiesa, il quale descrive l'impegno di un'antimafia declinata al femminile (2006); Umberto Santino e Anna Puglisi, che offrono un profilo storico dei ruoli delle donne in Cosa nostra (2005); Franca Imbergamo, pm antimafia, che scrive sull'esperienza delle donne del digiuno di PalermoLanzada, 1996; Lanzada, 2011); il regista Piero Li Donni che racconta il suo incontro con Marta Cimino, ideatrice del comitato dei lenzuoli; la scrittrice Rosella Postorino, autrice di un romanzo "L'estate che perdemmo Dio", in cui la giovane protagonista scrive lettere appassionate a Cesare Casella (1990).

Nell'ambito della ricerca sociale, inoltre, la sociologa Alessandra Dino si concentra sul potere della parola e del racconto, che accompagna il percorso di fuoriuscita delle 
donne dai contesti mafiosi (Dino, 2006), mentre la sociologa Ombretta Ingranscì delinea un ritratto di Rita Di Giovine, collaboratrice di giustizia (Ingrasci' 2007; 2013)) Concludendo, si può affermare che l'indagine sull'antimafia è intessuta di soggettività politiche femminili e che l'esperienza femminile, maturata in seno ad associazioni, che fin dagli inizi hanno interpretato e combattuto le mafie adottando un'ottica antigiustizialista, improntata all'antimafia sociale e culturale, ha ridisegnato con linguaggi creativi un nuovo immaginario dell'antimafia. Ciò è possibile coltivando la passione per la ricerca e la pratica di un sapere altro: quello di genere. I testi, gli articoli e le ricerche utilizzati per questo articolo provengono in gran parte dagli studi di genere e dalla cultura femminista, che ha modificato lo sguardo sulle tante battaglie per affermare la cultura della legalità.

\section{Se l'antimafia è donna (Sdisonorate, 2014)}

La storia dell'antimafia è fondamentalmente una storia maschile. Lo è quella ufficiale. Che si nutre degli elenchi dei protagonisti, degli eroi e delle vittime. Lo è quella della memoria popolare, fatta di volti, parole, leggende, ballate e cippi. Ed è comprensibile. Nella società italiana e ancor più marcatamente nell'Italia meridionale i ruoli di responsabilità istituzionale, come i ruoli di direzione politica e sindacale o di leadership civile sono stati tradizionalmente esercitati da maschi. Maschio per definizione il mafioso, rappresentante per antonomasia di una società-stato sessista che non ammette la donna nei ranghi dell'unica organizzazione che distribuisce la risorsa pregiata dell'onore. Maschio l'antimafioso, esponente di uno Stato o di una società in contrasto radicale con l'universo dei valori e dei comportamenti mafiosi. Maschi i sindacalisti del dopoguerra, maschi i magistrati e gli altri rappresentanti dello Stato degli anni Ottanta e Novanta.

Tutti simboli e sintesi di un movimento di idee dalle radici profonde (Dino, Callari, a cura di, 20090. Questo quadro non viene cambiato nella sua poderosa forza statistica dal fatto che esistano figure istituzionali femminili vittime della violenza mafiosa in tempi abbastanza recenti. Si pensi, con specifico riferimento alla lotta a Cosa Nostra, a Francesca Morvillo, moglie di Giovanni Falcone e anche lei magistrato, dilaniata con il marito nella strage di Capaci. O a Emanuela Loi, la poliziotta di scorta a Paolo Borsellino, la prima donna a cadere nella storia delle forze dell'ordine, il 19 luglio del 1992. Oppure, spaziando indietro nell'aspra vicenda nazionale della lotta per la legalità, si pensi a Renata Fonte, la giovane assessore alla cultura di Nardò, provincia di Lecce, uccisa nel 1984 per il suo impegno contro la speculazione edilizia sul parco naturale di Porto Selvaggio. II quadro inizia a cambiare, invece, e anche in misura significativa, se si considerano altri processi e fenomeni che con la lotta alla mafia hanno molto a che fare, e anzi ne sono parte integrante. Il primo è quello delle testimonianze di vita femminili ( Chirico, 2013). Anch'esse simboliche, nate al fianco di eroismi maschili, ma capaci di assumere nel tempo una loro potente autonomia di significati e di linguaggi. Ne è un esempio Francesca Serio, la madre del sindacalista Salvatore Carnevale, che rompendo un'omertà secolare ebbe il coraggio di denunciare alla giustizia gli assassini del figlio avendo solo la certezza morale delle loro responsabilità. $E$ che avviò così l'unico processo giudiziario nella storia d'Italia in cui nella veste di difensori si siano trovati su parti opposte, sia pure in gradi diversi del giudizio, due futuri presidenti della Repubblica (Sandro Pertini dalla parte della vittima, Giovanni Leone dalla parte degli imputati). Oppure Felicia Impastato, madre di Peppino, moglie di un mafioso e madre capace di allevare un figlio anti-mafioso. 
Esiste inoltre un processo ampio, articolato, diversamente presente nello spazio, che passa per alti e bassi mantenendo tuttavia una apprezzabile continuità e che ha come protagonista il mondo della scuola. Da Milano a Palermo, da Reggio Emilia a Catania, è cresciuto un lavoro di sensibilizzazione sul tema della mafia e di educazione alla legalità al cui centro sta - in modo del tutto tipico - la figura femminile dell'insegnante (Fiandaca, 1997). Migliaia e migliaia di insegnanti donne, ben al di là delle proporzioni implicite nel grado di femminilizzazione della professione. Generazioni di insegnanti donne il cui lavoro purtroppo non è mai stato sufficientemente stimato nelle sue dimensioni né nelle sue conseguenze civili. $̇$ un lavoro che adotta stili e modelli pedagogici differenti, che si avvale del sostegno finanziario di leggi regionali o si svolge del tutto gratuitamente. Ma che esprime lo sforzo di garantire verso le nuove generazioni quel lavoro di educazione che le altre istituzioni sociali e civili non garantiscono. $\dot{E}$ come se migliaia di donne trasferissero in una gigantesca dimensione collettiva la classica funzione di riproduzione da loro già svolta tra le mura domestiche.

Una funzione che, nei luoghi cosiddetti di trincea, contrasta direttamente l'analoga funzione svolta, con valori opposti, dalla donna nelle famiglie dell'universo mafioso. $E$ che nella società italiana in generale svolge invece una decisiva funzione di supplenza rispetto alle assenze (e alle ignavie) della politica, dei mass media e delle altre agenzie educative. I risultati di questo impegno si colgono senz'altro nella crescita di movimenti studenteschi di tipo nuovo (ad esempio "l'esercito dei sedicenni" degli anni novanta)

ma anche nelle disposizioni culturali con cui i giovani si orientano verso le professioni legali, nella nascita di un nuovo volontariato civile, nella maggiore consapevolezza sociale della gravità del problema dei poteri criminali.

Vi è poi una terza decisiva funzione assicurata dalla componente femminile alla lotta alla mafia. Ed è quella della partecipazione di base. Una partecipazione diffusa. Che non va affatto ridotta a funzione subalterna verso leadership maschili, perché si intreccia con (ed elabora) forme inedite di protagonismo femminile. La partecipazione implica infatti comunque una scelta di stare allinterno di un processo di sensibilizzazione e di riqualificazione dello spirito pubblico, di sostenere aprendo propri fronti di conflitto e competizione culturale - chi rappresenta lo Stato di diritto nei suoi punti più esposti. Implica la creazione di comitati, luoghi, simboli, linguaggi e opportunità di discussione. L'antimafia mostra così il suo volto prevalentemente femminile, oltre che nella nuova funzione civile della scuola, anche nella propria cittadinanza di riferimento: nelle presentazioni dei libri, nel pubblico dei lettori e dei telespettatori, nelle voci che orientano comunità parrocchiali e biblioteche, negli interessi che animano corsi e seminari universitari. Se l'impegno di riproduzione culturale è un fondamentale retroterra nella lotta alla mafia, quello della partecipazione diffusa ne è il terreno quotidiano. E i due impegni, come è ovvio, si aprono uno all'altro, contagiandosi ripetutamente in quello che è a pieno titolo un processo poliedrico di civilizzazione culturale. Spesso sperimentando il meccanismo della "doppia presenza" femminile, specie delle insegnanti, in entrambi i fronti(Fiandaca, op. cit. 1997). Si tratta, vorrei aggiungere, di Donne in campo. Ma vi è infine il fenomeno, più nuovo, del protagonismo diretto nella lotta alla mafia. Ossia della presenza femminile alla testa dei movimenti antimafia, una presenza che non nasce da rapporti affettivi, familiari, con le vittime della violenza mafiosa, ossia dalla spinta a trasformare il dolore in testimonianza. Ma da una scelta di campo che molto deve, naturalmente, alle stesse attività di testimonianza, come anche a quelle di riproduzione culturale e di partecipazione diffusa, dalle quali viene sospinta e 
alimentata (Modica, 2012). Basti pensare alle più note figure di sindaci donne in Sicilia nella stagione degli anni Novanta, come Maria Maniscalco a San Giuseppe Jato e Gigia Cannizzo a Partinico.

Oppure alla progressiva assunzione di responsabilità investigative da parte di magistrati donne, da Ilda Boccassini, che va volontaria a Caltanissetta dopo le stragi del '92 ad Anna Canepa, giudice ragazzina genovese in Sicilia, che viene allontanata prudenzialmente dall'isola nello stesso periodo per continuare il proprio impegno nella città di origine con spirito di pioniera. $O$ al ruolo cruciale di magistrate palermitane come Teresa Principato o Franca Imbergamo (solo per citare due tra le più conosciute).

D'altronde la stessa recente crescita di un'opposizione alla mafia e alle organizzazioni mafiose al nord molto deve alle figure femminili presenti nelle amministrazioni locali. È una donna, Maria Ferrucci, che si candida a sindaco di Corsico - ossia nel cuore dell'hinterland sud milanese colonizzato dai clan calabresi - con la parola d'ordine della lotta alla mafia e vince nel 2010. È una donna, Lucrezia Ricchiuti, consigliere comunale a Desio, a condurre una lotta serrata e quasi in solitudine contro l'intreccio politica-'ndrangheta in uno dei centri più inquinati della Brianza fino a ottenere, anche grazie alle inchieste giudiziarie successive, l'autoscioglimento del consiglio comunale cittadino e diventare poi vicesindaco di una nuova amministrazione dal chiaro indirizzo antimafioso. È un'altra donna, Donatella Albani, a ingaggiare nella sua veste di consigliere comunale di Bordighera, una lotta impossibile e di nuovo quasi solitaria contro il dominio costruito dai clan sul ponente ligure, finché le sue ragioni non diventano storia con lo scioglimento del consiglio comunale da parte del ministero dell'Interno. È ancora una donna, Beatrice Uguccioni, presidente del consiglio di zona 9 di Milano, a guidare una grande mobilitazione dei cittadini dopo l'incendio di una struttura sportiva comunale avvenuto in pieno giorno ad Affori.

Sono esempi che parlano per un mondo più ampio di amministratrici e professioniste, di intellettuali e giornaliste, tra cui va ricordata almeno la figura di Rosaria Capacchione, cronista del «Mattino» di Napoli. E a questo mondo va aggiunto quello - piccolo, particolare ma oltremodo significativo - delle donne che hanno rotto e rompono dall'interno dei clan l'omertà e la complicità mafiose. Donne che trapassano il senso di giustizia, dal pathos all'ethos. Va colto, peraltro, come queste dinamiche si inscrivano in una tendenza che appare ancora più generale. Le donne sembrano cioè sintonizzarsi con più naturalezza sulla frequenza della legalità. Basti pensare al peso crescente da loro assunto proprio nella magistratura, in cui costituiscono ormai la maggioranza delle nuove leve. Oppure anche al ruolo da loro giocato nei movimenti per la legalità dei primi anni Duemila, ossia nei famosi "girotondi" passati alla storia nel segno di nomi maschili ma che furono, in realtà, il frutto della fantasia, dell'indignazione, della diffusa capacità di leadership soprattutto di donne. Come spiegare questo divario di sensibilità di genere riscontrabile almeno nelle élites?

Probabilmente si combinano fattori diversi. Senz'altro pesano la minore partecipazione femminile alla vita e alle mediazioni culturali dei partiti e la maggiore distanza della donna dai meccanismi del potere (Albera, Blok, Bromberger, 2001). Ma contano, altrettanto probabilmente, fattori più profondi: dalla maggiore capacità femminile di dare forza politica ai sentimenti (si ricordino le donne di Plaza de Mayo) fino a una adesione più intensamente etica ai princìpi di eguaglianza e di giustizia, radicata in (e nutrita $\mathrm{da}$ ) una storia collettiva di disuguaglianza e 
discriminazioni. Più in generale si può avanzare la tesi che la marcia delle donne (della loro componente più avanzata, si intende) sul sentiero della legalità esprima ormai l'ingresso sulla scena sociale di un "popolo nuovo". Sia nelle teorie storicodemografiche sia in quelle socio-organizzative la nascita e l'affermazione di nuove popolazioni o popoli coincide con il declino di certi valori o tecnologie e con l'avanzata di altri ( Longrigg, 1997). Così nella società italiana la componente femminile, discriminata in misura paradigmatica e anzi unica in occidente dal codice penale, dai costumi o dalle stesse logiche della rappresentanza politica, può essere effettivamente vissuta, più che altrove, come un'identità collettiva nuova e diversa, complessivamente portatrice di un'altra moralità media. Senza indulgere a idealizzazioni improprie, sembra questa l'ipotesi esplicativa più interessante (anche se non esclusiva) di un fenomeno empirico che è comunque sotto gli occhi di qualsiasi osservatore appena attento: l'antimafia oggi è soprattutto donna. Dalla coraggiosa testimonianza di un dolore ribelle si è passati alla promozione consapevole di una più alta etica pubblica. Dal pathos della tragedia greca all'ethos della democrazia (Dino, 2011).

\section{Bibliografia}

AA.VV., Fiandaca G., (a cura di) Donne e mafie. II ruolo delle donne nelle organizzazioni criminali, Palermo, Università degli Studi di Palermo, Dipartimento di Scienze Penalistiche e Criminologiche, 2003.

AA.VV., Dal materno al mafioso. Ruoli delle donne nella cultura delle mafie, Quaderni di CLD, Firenze 1996.

AA.V., Donne di Mafia, Rivista Meridiana, Viella, n.66, 2010. Abbate L., Fimmine Ribelli. Le donne salveranno il paese dalla 'ndrangheta, Rizzoli, Milano, 2013.

Abbruzzese S., La paura in Occhiogrosso F., Ragazzi della Mafia, Franco Angeli, Milano, 1993.

Accati L., II marito della Santa. Ruolo paterno, Ruolo materno e la politica italiana, Meridiana,13, 1992.

Aiello P., Lucentini U., Mafia detta Mafia, San Paolo Edizioni, Milano, 2012.

Albera D., Blok A., The mediterraneansas a field of ethnologicalstudy. A retrospective, in Albera D., Blok A., Bromberger C. (a cura di), L'anthropologie de la Mèditerranée, Maisonneuve\&Larose, Paris, 2001.

Bartolotta Impastato F., (a cura di Puglisi A.) La mafia in casa mia, La Luna, Palermo,1987.

Bolzani O., (a cura di) L'emancipazione malata. Sguardi femministi sul lavoro che cambia, Libera Università, Milano 2010.

Cerati C. La storia vera di Carmela che si ribella all'oblio e combatte la mafia, Marsilio, Venezia, 2009.

Chinici C., Santino U., La violenza programmata, Franco Angeli, Milano, 1991.

Chirico D., Magro A., Dimenticati. La storia e le storie delle donne e degli uomini assassinati dall'organizzazione criminale più segreta e potente del mondo, Castelvecchi, Roma, 2010. 
Chirico F., lo parlo, donne ribelli in terra di 'ndrangheta, Castelvecchi, Roma, 2013.

Costantino C., Roberta Lanzino, ragazza, Round Robin, Roma, 2012.

Dalla Chiesa N., Le ribelli, Melampo, Milano 2006,

De Maria M., La scelta di Lea, Melampo, Milano, 2013.

Canale Rosy., Zuccalà E. La mia 'ndrangheta, Paoline, Milano 2012.

Di Lorenzo S.; La grande madre mafia. Psicoanalisi del potere mafioso, Pratiche, Parma, 1996.

Di Maria F., Lavanco G., Mafia e codici familiari. L'ombra della madre, in Psicologia Contemporanea, n. 155, settembre/ottobre, 1999.

Dino A., Coscienza e potere. Narrazioni attraverso il mito, (a cura di e in collaborazione con L. Callari), Mimesis Edizioni, Milano, 2009.

Dino A., Donne, mafia e processi di comunicazione, in "Rassegna Italiana di Sociologia", anno XXXIX, n. 4, 1998.

Dino A., Criminalità dei potenti e metodo mafioso, (a cura di), Milano, Mimesis Edizioni, 2009.

Dino A., Donne di Cosa Nostra, "Nuove Effemeridi", anno XIII, n. 50, 2000b/II, pp. 74-91.

Dino A., La mafia devota. Chiesa, religione, Cosa Nostra, RomaBari, Editore Laterza, 2008.

Dino A., La violenza tollerata. Mafia, poteri, disobbedienza, (a cura di), Mimesis Edizioni, Milano2006.

Dino A., Mutazioni. Etnografia del mondo di Cosa Nostra, Palermo, Edizioni La Zisa, 2002.

Dino A., Pentiti. I collaboratori di giustizia, le istituzioni, l'opinione pubblica (a cura di), Roma, Donzelli Editore, 2006.

Dino A., Poteri criminali e crisi della democrazia (a cura di), Milano, Mimesis, 2011.

Dino A., Principato T. Mafia donna. Le vestali del sacro e dell'onore, Flaccovio, Palermo 1997.

Dino A., Sistemi criminali e metodo mafioso, (a cura di e in collaborazione con L. Pepino), FrancoAngeli, Milano, 2008.

Ebano G., Felicia e le sue sorelle. Dal secondo dopoguerra alle stragi del '92-93: venti storie di donne contro la mafia, Ediesse 2005

Fallucca P. Leggi altre, in "Mezzocielo", Marzo 1993.

Fiandaca G., La discriminazione sessuale tra paradigmi giudiziari e paradigmi culturali, in "Segno", XXIII, 1997.

Fiume G., Ci sono donne nella mafia?, in "Meridiana", 7-8, 198990.

Gambetta D., La mafia siciliana. Un'industria della protezione privata, Einaudi, Torino 1992.

Graziosi M., Infirmitassexus. La donna nell'immaginario penalistico, in Democrazia e diritto, n. 2, 1993. 
Gribaudi G., Donne di Camorra e identità di Genere in Meridiana, n.67, 2007.

Incandela F., Donne di mafia. Donne contro la mafia, Libridine, 2007.

Ingrascì O., Le donne della 'Ndrangheta: il caso Serraino-Di Giovine, pp. 46-51, in Ingrasci O., Le donne in cosa nostra e nella 'ndrangheta, in Ciconte E., Forgione F.,Sales I., Nuovo Atlante delle Mafie, Rubbettino Soveria Manelli, 2013.

Ingrasci O., Storie di Mafia al femminile, Bruno Mondadori, Milano, 2007.

Lanza A., Donne contro la mafia. L'esperienza del digiuno a Palermo, Datanews, Roma 1994.

Lanza A.,( a cura di) Ho fame di giustizia, Navarra, Marsala, 2011.

Lo Verso G., La mafia dentro: psicopatologia di un fondamentalismo, Franco Angeli, Milano 2005.

Longrigg C., L'altra faccia della mafia, Ponte alle Grazie, Milano 1997.

Madeo L., Donna e mafia, Mondadori, Milano 1994.

Madeo L., Donne di mafia: vittime, complici e protagoniste, Mondadori, 1994.

Maraini D., Sulla Mafia, Perrone, 2009.

Modica G., ( a cura di) Contro la mafia perché donne, aprilemaggio, 2012.

Morini C., Per amore o per forza. Femminilizzazione del lavoro e biopolitiche del corpo, Ombre Corte, Verona, 2010.

Pizzini V., Gambetta D., Gender Norms in the Sicilian Mafia 1945 -1986, in M.L. Arnot e C. Usborne (eds), Gender and Crime in Modern Europe, U.C.L, London 1999.

Pasculli A., II ruolo della donna nelle organizzazioni criminali, Rivista di Criminologia Vittimologia e Sicurezza Vol. III - N. 2, Maggio-Agosto, 2009.

Postorino R., L'estate che perdemmo Dio, Einaudi, Torino, 2009. Puglisi A., Donne, mafia e antimafia, Trapani, Di Girolamo, 2005.

Puglisi A., Sole contro la mafia, La Luna, Palermo 1990.

Reski Petra, Rita Atria : la picciridda dell'antimafia, Nuovi Mondi, Modena, 2011.

Rizza S., Una ragazza contro la mafia. Rita Atria, morte per solitudine, La Luna, Palermo 1993.

Scarfò A; Zagaria C. Malanova, Sperling \& Kupfer, Milano, 2010.

Sdisonorate, Reportage 1 e 2, reperibile su www.terrelibere.org 2014

Siebert R., Le donne, La mafia, II Saggiatore, Milano, 1994.

Siebert R., Mafia e quotidianità, II Saggiatore, Milano 1996.

Siebert R., Storia di Elisabetta, Editore Pratiche, 2001.

Siebert R. Donne di mafia: affermazione di uno pseudo-soggetto . Il caso della 'ndrangheta in II ruolo delle donne nelle organizzazioni criminali, Palermo,Università degli Studi di Palermo, Dipartimento di Scienze Penalistiche e Criminologiche, 2003.

Vitale G., Costanzo C., Ero cosa Loro, Milano, Mondadori, 2009. 